\title{
Music Perception Abilities and Ambiguous Word Learning: Is There Cross-Domain Transfer in Nonmusicians?
}

\author{
Eline A. Smit ${ }^{1,2 *}$, Andrew J. Milne ${ }^{1}$ and Paola Escudero ${ }^{1,2}$ \\ 'The MARCS Institute for Brain, Behaviour and Development, Western Sydney University, Sydney, NSW, Australia, \\ ${ }^{2} A R C$ Centre of Excellence for the Dynamics of Language, Canberra, ACT, Australia
}

OPEN ACCESS

Edited by:

Caicai Zhang,

The Hong Kong Polytechnic University, Hong Kong SAR, China

Reviewed by:

Francesca Talamini, University of Padua, Italy

Mireille Besson,

UMR7291 Laboratoire de Neurosciences Cognitives (LNC), France

*Correspondence: Eline A. Smit e.smit@westernsydney.edu.au

Specialty section: This article was submitted to

Language Sciences, a section of the journal

Frontiers in Psychology

Received: 25 October 2021 Accepted: 08 February 2022 Published: 28 February 2022

Citation:

Smit EA, Milne AJ and Escudero P (2022) Music Perception

Abilities and Ambiguous Word Learning: Is There Cross-Domain

Transfer in Nonmusicians?

Front. Psychol. 13:801263.

doi: 10.3389/fpsyg.2022.801263
Perception of music and speech is based on similar auditory skills, and it is often suggested that those with enhanced music perception skills may perceive and learn novel words more easily. The current study tested whether music perception abilities are associated with novel word learning in an ambiguous learning scenario. Using a cross-situational word learning (CSWL) task, nonmusician adults were exposed to word-object pairings between eight novel words and visual referents. Novel words were either non-minimal pairs differing in all sounds or minimal pairs differing in their initial consonant or vowel. In order to be successful in this task, learners need to be able to correctly encode the phonological details of the novel words and have sufficient auditory working memory to remember the correct word-object pairings. Using the Mistuning Perception Test (MPT) and the Melodic Discrimination Test (MDT), we measured learners' pitch perception and auditory working memory. We predicted that those with higher MPT and MDT values would perform better in the CSWL task and in particular for novel words with high phonological overlap (i.e., minimal pairs). We found that higher musical perception skills led to higher accuracy for non-minimal pairs and minimal pairs differing in their initial consonant. Interestingly, this was not the case for vowel minimal pairs. We discuss the results in relation to theories of second language word learning such as the Second Language Perception model (L2LP).

Keywords: music perception, pitch, phonological processing, cross-situational word learning, auditory perception

\section{INTRODUCTION}

Music and language are universal to humans (Patel, 2003) and the connection between the two has been an object of research for centuries, with early ideas even suggesting that music is a spin-off of language in evolution (Pinker, 1997). While the precise origins of music and language remain unclear, there are many parallels that can be drawn between the two. Both use a rule-based hierarchical structure organized into discrete elements and sequences (Tervaniemi et al., 1999; Tervaniemi, 2001; Patel, 2003; Degé and Schwarzer, 2011; Burnham et al., 2015), such as syllables, words, and sentences for language and single notes, intervals, chords, and musical phrases for music (Ong et al., 2016). When focusing on the acoustic characteristics 
of music and speech sounds, similarities can be found in the reliance on segments of rhythm and harmony alternated with silence, pitch, acoustic envelope, duration, and fundamental frequency (Varnet et al., 2015). In order to understand music and speech, a listener needs to categorize sounds into meaningful units. For speech, perceptual skills are needed to distinguish sounds into separate vowels or consonants and for music into pitches (Hallam, 2017). The auditory skills needed to process language are similar to those needed to discriminate between rhythms (Lamb and Gregory, 1993), harmonies, and melodies (Barwick et al., 1989; Lamb and Gregory, 1993; Anvari et al., 2002). Numerous studies support the overlap of auditory processes involved in music and speech perception (Overy, 2003; Tallal and Gaab, 2006; Patel and Iversen, 2007; Sammler et al., 2007; Wong and Perrachione, 2007; Chandrasekaran et al., 2009; Kraus and Chandrasekaran, 2010; Besson et al., 2011; Rogalsky et al., 2011; Schulze et al., 2011; Bidelman et al., 2013; Gordon et al., 2015; Kraus and White-Schwoch, 2017) and individuals with musical training appear to be advantaged in these shared processes (Krishnan et al., 2005; Bigand and Poulin-Charronnat, 2006; Krizman et al., 2012; White-Schwoch et al., 2013; Elmer et al., 2014).

Those that are expert listeners in either music or language have been found to show cross-domain transfer (Ong et al., 2016), where an advantage is found for perception in the other domain; for example, in word segmentation (François et al., 2013), syllabic perception (Musacchia et al., 2007; Ott et al., 2011; Elmer et al., 2012; Kühnis et al., 2013; Chobert et al., 2014; Bidelman and Alain, 2015), receptive and productive phonological skills at the word, sentence and passage level (Slevc and Miyake, 2006), and word dictation (Talamini et al., 2018). It is suggested that long-term expertise in music, which is gained by years of practice, has led to a fine-tuning of the auditory system (Strait and Kraus, 2011a,b), as evidenced by enhanced neural responses to changes in acoustic elements, such as pitch, intensity, and voice onset time (Schön et al., 2004; Magne et al., 2006; Jentschke and Koelsch, 2009; Marie et al., 2011a,b). Musicians indeed show enhanced cortical processing of pitch in speech compared to nonmusicians (Magne et al., 2006; Besson et al., 2007; Musacchia et al., 2007; Kraus and Chandrasekaran, 2010). These and numerous other studies support the idea of cross-domain transfer between music and speech perception (see Hallam, 2017 for an extensive list). The present study focuses on the potential auditory processing advantages in pitch perception and auditory working memory (Ott et al., 2011; Kühnis et al., 2013; Pinheiro et al., 2015; Dittinger et al., 2016, 2017, 2019) associated with music perception skills. Many examples of the effect of music training on speech processing have been reported. For instance, training in music has been associated with phonological perception in the native language (L1; Zuk et al., 2013) and with fluency in a second language (L2; Swaminathan and Gopinath, 2013; Yang et al., 2014). As well, longitudinal studies in children's speech perception found positive effects of music training (Moreno et al., 2009; Degé and Schwarzer, 2011; François et al., 2013; Thomson et al., 2013). Regarding the transfer of music experience to word learning, Dittinger et al. $(2016,2017,2019)$ presented listeners with unfamiliar Thai monosyllabic words and familiar visual referents during a learning phase and tested them on their ability to match the words with their corresponding visual objects. Overall, they found that both music training led to higher accuracy in both young adults and children. Additionally, a longitudinal effect of music training was shown, as musicians had the same advantage when tested 5 months later (Dittinger et al., 2016).

However, counter-examples to a positive association between music training and speech perception also exist (Ruggles et al., 2014; Boebinger et al., 2015; Swaminathan and Schellenberg, 2017; Stewart and Pittman, 2021). For instance, Swaminathan and Schellenberg (2017) found that rhythm perception skills predicted English listeners' discrimination of Zulu phonemic contrasts, but only for contrasts that closely resembled English phonemic contrasts. The authors found no association between other music perception skills, such as melody perception or general music training and non-native speech perception, suggesting that an effect of rhythm rather than pitch is related to participants' native language background rather than their music skills. Specifically, unlike for tonal languages, English does not contrast pitch for signaling lexical meaning; hence, it is likely that listeners focus on other cues, such as temporal cues, to distinguish one word from another.

Apart from the ability to perceive novel or familiar phonological contrasts, another important component involved in speech processing, including novel word learning, is working memory. Working memory, which is a short-term memory involved in immediate conscious perceptual and linguistic processing, plays an important role in novel word learning (Gathercole et al., 1997; Warmington et al., 2019). Mixed results have been found regarding a musician's advantage in working memory, with some studies finding no difference between musicians and nonmusicians (Hansen et al., 2012), whereas others find improved auditory and verbal working memory for musicians compared to nonmusicians (Parbery-Clark et al., 2011; Bergman Nutley et al., 2014). A meta-analysis conducted by Talamini et al. (2017) on different types of memory found a medium effect size for short-term and working memory with musicians performing better than nonmusicians, depending on the type of stimulus used.

Most studies examining the link between speech processing and musical abilities have compared professional musicians to nonmusicians (see Zhu et al., 2021), with a large focus on explicit tasks when comparing linguistic and musical abilities (e.g., Dittinger et al., 2016, 2017, 2019). In such tasks, there is no ambiguity during learning, but the link between words and meaning in daily life is much more ambiguous without immediate clear connections, with studies showing that pairing between words and their referent objects are learned by tracking co-occurrences through repeated exposure (e.g., Smith and Yu, 2008; Escudero et al., 2016b; Mulak et al., 2019). Very little is known about the role of musical abilities for ambiguous word learning scenarios, which are most common in everyday life of word learning (Tuninetti et al., 2020). In the realm of music perception, recent studies have shown that musical elements, such as musical grammar 
(Loui et al., 2010), harmony (Jonaitis and Saffran, 2009), musical expectation (Pearce et al., 2010), and novel pitch distributions from unfamiliar musical scales (Ong et al., 2017a; Leung and Dean, 2018), can be learned through statistical learning. Statistical learning is a domain-general learning mechanism leading to the acquisition of statistical regularities in (in this case auditory) input. This type of learning may lead to cross-domain transfer between music and language due to learners showing sensitivity toward particular acoustic cues (e.g., pitch; Ong et al., 2016) which may result in improved ambiguous word learning. Despite the potential effect of music abilities on ambiguous word learning and the many types of learners considered in statistical word learning studies (such as young infants, children and adults, and L2 learners Yu and Smith, 2007; Smith and Yu, 2008; Suanda et al., 2014; Escudero et al., 2016b,c; Mulak et al., 2019), participants' musical experience or expertise have yet to investigated. In sum, it has been established that music and language rely on similar general auditory processing skills and, although results are mixed, the majority of studies finds an advantage for music training on auditory and speech perception. By testing whether music abilities in a nonmusician population can help ambiguous word learning, we can further unravel more influences of music on language learning than previously shown.

The current study tests the effect of specific music perception abilities on statistical learning of novel words in a nonmusician adult population. We tested musical abilities through two adaptive psychometric tests targeting specific music perception skills, namely, the ability to perceive fine-pitch mistuning, through the Mistuning Perception Test (MPT; Larrouy-Maestri et al., 2018, 2019), and the ability to discriminate between pitch sequences, through the Melodic Discrimination Test (MDT; Harrison et al., 2017; Harrison and Müllensiefen, 2018). The MPT is an adaptive psychometric test measuring sensitivity to intonation accuracy in vocal musical performance (Larrouy-Maestri et al., 2018, 2019). Perception of vocal mistuning is a core musical ability, as evidenced by its high correlation with other musical traits (Law and Zentner, 2012; Kunert et al., 2016; Larrouy-Maestri et al., 2019), and its importance when judging the quality of a musical performance (Larrouy-Maestri et al., 2019). The MDT aims to test melodic working memory, as it requires melodies to be held in auditory working memory in order for participants to compare and discriminate them correctly (Dowling, 1978; Harrison et al., 2017; Harrison and Müllensiefen, 2018). To do well in these tasks, specific auditory processing skills, in particular pitch perception and auditory working memory, are required. A recent large-scale study across thousands of speakers of tonal, pitch-accented, and non-tonal languages using these two tasks (and a beat alignment task) has shown that language experience shapes music perception ability (Liu et al., 2021). Here, we test the opposite, namely, whether the same music perception skills help with language learning, and specifically when learning novel words with different degrees of phonological overlap. Our specific focus is on pitch processing abilities but acknowledge that rhythm processing is also an important component in music and language processing (see Swaminathan and Schellenberg, 2017).

To test whether pitch perception and auditory working memory are helpful when learning words in ambiguous scenarios, we used a cross-situational word learning (CSWL) paradigm in which meanings of new words are learned through multiple exposures over time without explicit instruction, where learning of word-object pairings can only take place through their statistical co-occurrences (e.g., Escudero et al., under review; Yu and Smith, 2007; Kachergis et al., 2010; Smith and Smith, 2012; Escudero et al., 2016a,b, 2021; Mulak et al., 2019; Tuninetti et al., 2020). Early CSWL experiments focused on words with very little phonological overlap (e.g., Smith and Yu, 2008; Vlach and Johnson, 2013), where a listener can rely on other cues to learn the novel words and does not have to focus on the fine phonological details of each word (Escudero et al., 2016b). Therefore, (Escudero et al., 2016a,b) and Mulak et al. (2019) studied CSWL of monosyllabic non-minimal and minimal pairs, differing only in one vowel or consonant, to test whether listeners can encode sufficient phonological detail in a short time to learn these difficult phonological contrasts. It was found that accurate phonological encoding of vowel and consonant contrasts predicts high performance in CSWL tasks (Escudero et al., 2016a; Mulak et al., 2019).

In the present study, we thus tested whether musical ability impacts word learning of phonologically overlapping words using Escudero et al. (2016b) and Mulak et al. (2019)'s CSWL paradigm. Overall, we hypothesize that those with stronger musical abilities are better at perceiving speech sounds due to enhanced pitch perception and working memory, and that will be reflected in higher accuracy overall in the CSWL task. We may also see differences in how well vowels and consonants are learned, due to higher acoustic variability in vowels compared to consonants (Ong et al., 2015), which may favor learners with stronger pitch perception skills.

\section{MATERIALS AND METHODS}

\section{Participants}

Fifty-four participants took part in the study and were tested online, which is our common practice since the start of the COVID-19 pandemic, using our validated online testing protocols (Escudero et al., 2021). In Escudero et al. (2021), we compared online and face-to-face testing using the same CSWL design and online testing results were found to be very similar to results from the laboratory. Ten participants were excluded from the analysis due to technical difficulties, mostly internet dropouts during the experiment or excessive environmental noise, leading to a total participant sample of $44\left(M_{\text {age }}=26.79\right.$, $S D_{\text {age }}=11.12$, 33 females). Participants were recruited through the Western Sydney University's online research participation system (SONA) or via word-of-mouth and participation was rewarded with course credit for the former and voluntary for the latter. Written informed consent was obtained online from all participants prior to the start of the experiment, and the 
study was approved by the Western Sydney University Human Research Ethics Committee (H11022).

\section{Materials \\ Questionnaires}

The questionnaires conducted at the beginning of the experiment consisted of two parts: a language and a musical background questionnaire. The language background questionnaire consisted of questions aimed to get detailed information regarding participants native (and other) language, as well as the language background of their parents/caretakers. The musical background questionnaire is the Goldsmiths Musical Sophistication Index (GMSI; Müllensiefen et al., 2014), which aims to collect widerange data related to one's engagement with music (e.g., music listening and music performance behavior). Both questionnaires were administered through Qualtrics (Qualtrics, Provo, UT). From the GMSI, 23 participants indicated having zero years of experience with playing an instrument, and seven had 10 or more years of experience. From the language questionnaire, we found that 17 were Australian English monolinguals and 27 were bi- or multilinguals.

\section{Cross-Situational Word Learning}

All words and visual referents have been used in prior CSWL studies (Vlach and Sandhofer, 2014; Escudero et al., 2016a,b; Mulak et al., 2019; Escudero et al., under review). Novel words consisted of eight monosyllabic nonsense words recorded by a female native speaker of Australian English and followed a consonant-vowel-consonant (CVC) structure while adhering to English phonotactics. The stimuli were produced in infantdirected speech (IDS) as we are replicating previous studies that used IDS to compare adult and infant listeners and included two tokens for each word to match prosodic contours across all stimuli (Escudero et al., 2016a,b).

The eight words were combined into minimal pair sets to form specific consonant or vowel minimal pairs or non-minimal pairs. The two types of minimal pairs featured words that either differed in their initial consonant (consMPs; e.g., BON-TON) or in their vowel (vowelMPs; e.g., DIT-DUT). Non-minimal pairs were formed by pairing two words from each of the two minimal pair types in random order (nonMPs; e.g., BON-DIT).

Every novel word was randomly paired with a color picture of a novel item, which is not readily identifiable as a realworld object. These word-referent pairings were the same for all participants. An overview of the novel words and visual referents is presented in Figure 1.

\section{Mistuning Perception Test}

The MPT, which is an adaptive psychometric test, uses short excerpts (6-12s) of musical stimuli from pop music performances which are representative of real-life music and are therefore ecologically valid (from MedleyDB; Bittner et al., 2014). The test highly correlates with low- and high-level pitch perception abilities, such as pitch discrimination and melody discrimination, and thus provides an assessment of important pitch processing abilities

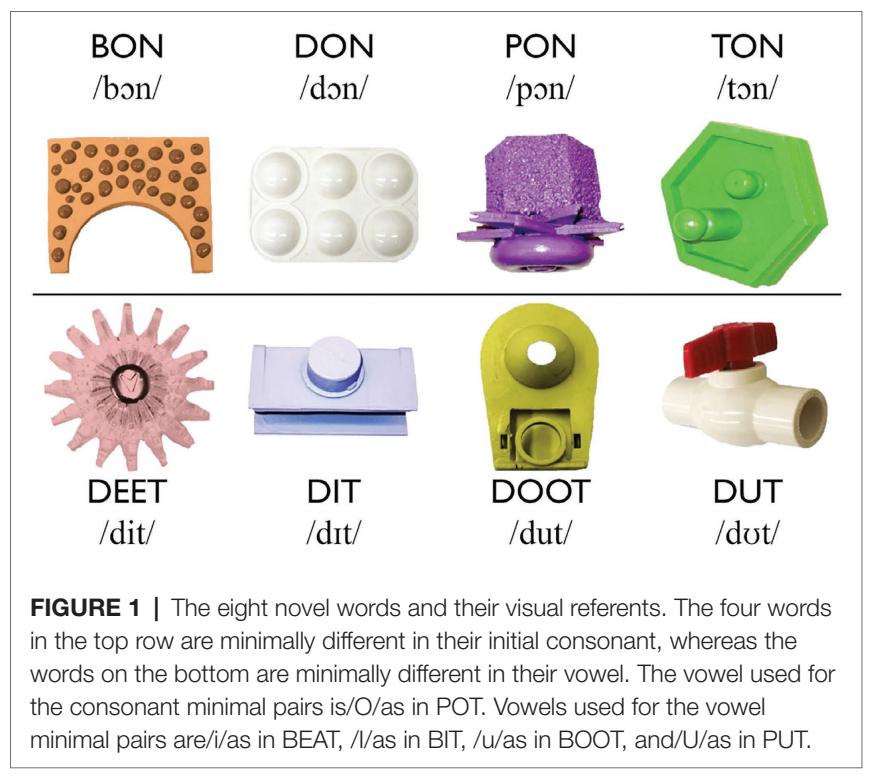

(Larrouy-Maestri et al., 2019). In a two-alternative forced-choice task, participants were presented with a pitch-shifted version (out-of-tune) and the normal version (in-tune) of a stimulus and were asked to indicate which version was out-of-tune. Pitch shifting varied from 10 cents to 100 cents, sharp, and flat (for more details about the construction of the MPT, see LarrouyMaestri et al., 2019). Before starting the task, participants received an example of an out-of-tune and an in-tune version. A demo of the experiment can be found on https://shiny.gold-msi.org/ longgold_demo/?test=MPT.

\section{Melodic Discrimination Test}

Similar to the MPT, the MDT is also an adaptive psychometric test. The MDT is developed to test one's ability to discriminate between two melodies (Harrison et al., 2017; Harrison and Müllensiefen, 2018). Participants are presented with a threealternative forced-choice (3-AFC) paradigm where they listen to three different versions of the same melody, each with a different pitch height (musical transposition), and with one containing an altered note produced by changing its relative pitch compared to the base melody (Harrison et al., 2017), resulting in a pitch height change for one note compared to the other melodies. Each melody can be altered using four pre-determined constraints: (1) melodies with five notes or fewer cannot have the first nor last note altered, (2) melodies with six notes or longer cannot have the first two nor last two notes altered, (3) the note cannot be altered by more than six semitones, and (4) the altered not must be between an eight note and a dotted half note in length (see Harrison et al., 2017). Participants are asked to indicate which of the three melodies are the odd one out. Participants heard an implementation of the MDT with 20 items (see doi:10.5281/ zenodo.1300951) using the shiny package in $\mathrm{R}$ (Chang et al., 2020) which uses an adaptive item selection procedure with each participant's performance level determining the level of difficulty of item presentation. Performance level is estimated 
using Item Response Theory (de Ayala, 2009). A demo of the experiment can be found on https://shiny.gold-msi.org/longgold_ demo/?test=MDT. Tests scores for both the MDT as the MPT are computed as intermediate and final abilities with weightedlikelihood estimation (Warm, 1989) and using Urry's rule for item selection (Magis and Raîche, 2012).

\section{Procedure}

We followed our adult online testing protocol, which was validated in Escudero et al. (2021), for details please see on https://osf.io/ nwr5d/. In short, participants signed up for a timeslot on SONA after which they received an email with specific instructions for the experiment (e.g., wearing headphones and participating from a silent study space with no background noise was required) and an invitation for a Zoom call. Participants unable to meet the participation requirements were excluded from the analysis (see Section "Participants"). During the Zoom call, participants were first familiarized with the procedure and then sent links to the consent forms, background questionnaires, and the experiment. During the experiment, they were asked to share their screen and computer audio throughout the entire video call, apart from when filling out the questionnaire to ensure privacy. Participants' screen and audio sharing enabled experimenter's verification of appropriate auditory stimuli presentation and participants' attention. The experimenter was on mute and with their video off during the experiment to avoid experimenter bias.

Participants first completed the language and musical background questionnaires and were then instructed to start the CSWL task. The CSWL task consisted of a learning and a test phase set up in PsychoPy 3 (Peirce, 2007; Peirce et al., 2019) hosted on Pavlovia.org. Following previous CSWL studies, minimal instruction was provided (i.e., "Please listen to the sounds and look at the images") prior to the learning phase. During the learning phase, participants saw 24 trials each consisting of two images accompanied by auditory representations of two words without indication of which word corresponded to which image. The visual referents were presented first for $0.5 \mathrm{~s}$ before the onset of the first word. Both words lasted for $1 \mathrm{~s}$ and were followed by a $0.5 \mathrm{~s}$ inter-stimuli interval (ISI). After this, a $2 \mathrm{~s}$ inter-trial interval (IT) consisting of a blank screen was then presented, leading to a total trial time of $5 \mathrm{~s}$. The learning phase was directly followed by a test phase of 24 trials, for which participants were told that they would be tested on what they have learned and to indicate their answers by pressing specific keys on the keyboard. Every test trial presented two possible visual referents simultaneously on the screen for $3 \mathrm{~s}$. During this, participants heard one spoken target word four times (with alternating tokens of the words) and were then asked to indicate which visual referent (the left or the right one) corresponded with the target word by pressing a key on the keyboard any time after the onset of the target word. Trial order was randomized across all participants. The presentation of left and right of the visual referents was counterbalanced and resulted in two between-subject learning conditions. A blank screen of $2 \mathrm{~s}$ was presented in between trials. Directly after the CSWL task, participants completed the MDT and the MPT task to measure their music perception abilities.

\section{STATISTICAL ANALYSIS}

We used a Bayesian Item Response Theory (IRT) model to analyze accuracy. IRT models are particularly useful for predicting the probability of an accurate answer depending on an item's difficulty, its discriminability, a participant's latent ability, and a specified guessing parameter (Bürkner, 2020), which provides a lower bound for the model's predictions. The statistical analyses were run in the statistical program R ( $\mathrm{R}$ Core Team, 2020) with the brms package using Stan (Bürkner, 2017, 2018; R Core Team, 2020).

We used approximate leave-one-out (LOO) cross-validation to find the model that generalizes best to out-of-sample data. Additionally including GMSI or participant's language background did not improve the out-of-sample predictions of the model.

The best model included only the interaction between Pair type and MPT. However, as we are interested in both MPT and MDT as main factors, we will report the next best model. The difference in the LOOIC values for these two models is negligible. Prior to fitting the models, we tested for correlation between MPT, MDT, and GMSI. MPT and MDT were moderately positively correlated, $r(1054)=0.39, p<0.005$; MPT and GMSI were moderately positively correlated, $r(1054)=0.30$; and MDT and GMSI were weakly positively correlated, $r(1054)=0.11$.

Accuracy was modeled as a binary response variable, with 0 for inaccurate and 1 for accurate. We used a 4-parameter non-linear logistic model (4PL, Agresti, 2010) on the Bernoulli distribution with an item, a person and a guessing parameter. The discriminability parameter is removed. The item parameter models the difficulty of the tested items (in this case the pair types); the person parameter models the individual ability of each participant. The guessing parameter represents the probability of being accurate if participants were only guessing (Bürkner, 2020). All of our trials are binary forced choice; hence, we use a fixed guessing parameter of 0.5 . An advantage of using IRT for modeling binary accuracy responses is that this probability can be taken into account as a type of baseline in the model, meaning that the model's estimates of the underlying probability of being correct will not fall below the 0.5 threshold. We did not include a discrimination parameter, as all tested items are very similar.

The categorical variable Pair type was turned into a factor and modeled using dummy coding, which is the default in R. For MPT and MDT, we are using the raw data scores, as recommended by the experiment designers (MPT: LarrouyMaestri et al., 2018, 2019; MDT: Harrison et al., 2017; Harrison and Müllensiefen, 2018), which were computed from the underlying item response models. These scores range from -4 to +4 . GMSI was scaled and centered to a previously determined population mean from Harrison and Müllensiefen (2018).

For the 3-PL IRT accuracy model, we included separate priors for the item, person and guessing parameters. As detailed below, all such priors were weakly informative in that they weakly favor an effect of zero size and disfavor unfeasibly large effects. The following model formula (including priors) was run in $\mathrm{R}$ : 


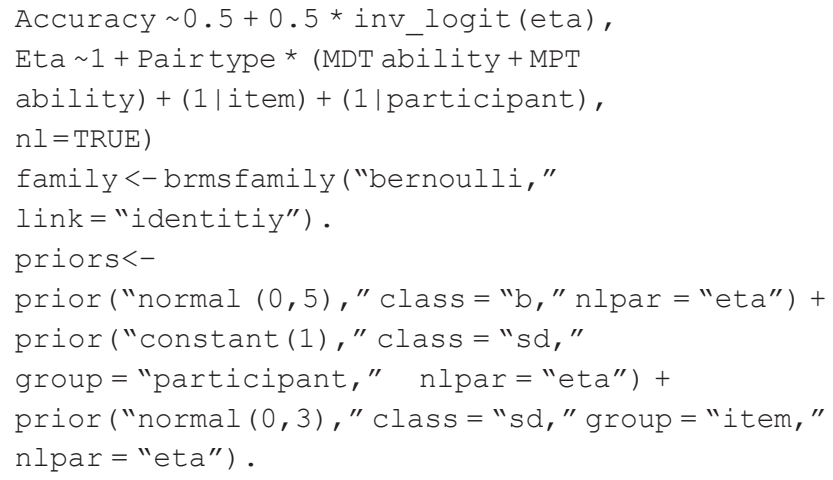

An important aspect of Bayesian regression is that it calculates the whole posterior distribution of each effect, which allows for the calculation of credibility intervals. In contrast with frequentist confidence intervals, credibility intervals indicate the $95 \%$ certainty that reported effect falls within the range of the interval (Smit et al., 2019). Evidence for a hypothesized effect will be assessed through evidence ratios, which quantify the likelihood of a tested hypothesis against its alternative (Bürkner, 2017, 2018). We consider evidence ratios of $>10$ to be strong evidence and above $>30$ to be very strong evidence [see Jeffreys (1998), as cited by Kruschke (2018)]. For directional hypotheses, where the predicted direction of an effect is given, effects with evidence ratios of $>19$ are roughly similar to an alpha of 0.05 in null-hypothesis significance testing (NHST; Makowski et al., 2019; Milne and Herff, 2020).

We expect that high musical perception abilities transfer to stronger phonological processing which subsequently translates to higher performance in the CSWL task (as evidenced by higher accuracy), compared to those with less musical perception abilities. With regards to the three tested pair types, we expect them to follow the same pattern as in previous CSWL studies, namely, a higher performance for nonMPs and consMPs and lower performance for vowelMPs (Escudero et al., 2016a). Additionally, we were interested in the differences between the moderations of MPT and MDT per pair type. As the MPT tests for perception of fine-pitch changes, one might expect participants with higher MPT scores to learn vowel contrasts more easily due to the acoustic similarities between musical pitch and vowels. As MDT measures auditory shortterm memory, we expect high MDT scores to positively correlate with accuracy in general.

\section{RESULTS}

Figure 2 shows the overall percentage of accurate responses per pair type. Performance across pair types appears to be very similar and participants were able to learn all pair types during the task, as evidence by performance being significantly above chance (see Figure 2). Accuracy for these learners is similar, albeit a little lower, to that found in a previous study (between 0.60 and 0.70 for all pair types) using the exact same design and online testing methodology (Escudero et al., 2021).
Hypothesis tests run on the results from the multilevel Bayesian model show strong evidence that for participants with average MDT and MPT, accuracy for consMPs is lower than for nonMPs (see Table 1, hypothesis 1). We did not find sufficient evidence to support a difference between the other pair types (hypotheses 2 and 3). We then tested whether performance per pair type is moderated by MPT and MDT ability. As shown in Figure 3, mean accuracy for nonMPs does not appear to be moderated by MPT ability, whereas for consMPs, higher MPT ability leads to higher accuracy, which was not expected. Also unexpectedly, the opposite occurs for vowelMPs, where higher MPT ability negatively impacts performance. As per our predictions, for MDT ability (see Figure 4), we see that higher scores generally lead to improved accuracy, especially for nonMPs and vowelMPs. However, important to note is that, as visualized by the colored ribbons in Figures 3, 4, the slopes' credibility intervals are highly overlapping, which indicates that the evidence for these differences might not be decisive. Therefore, we conducted hypothesis testing to confirm this (see hypotheses 4-6 for MPT ability and 10-12 for MDT ability in Table 1). As can be seen in Table 1, MDT ability influences accuracy in the expected direction (i.e., higher MDT leads to higher accuracy) for all pair types, but unexpectedly, MPT has a negative effect on accuracy for vowelMPs.

Regarding the extent to which the effect of MPT and MDT differs by pair type, unexpectedly, we find very strong evidence that MPT ability has a stronger impact on accuracy for consMPs than for nonMPs and vowelMPs (see Table 1; hypotheses 7 and 9) and strong evidence for nonMPs compared to vowelMPs (see Table 1; hypothesis 8). Thus, not only does MPT negatively influence the learning of vowelMPs as shown in hypothesis 4, but it also impacts the learning of vowelMPs less strongly than the learning of nonMPs and consMPs. Our finding of strong evidence suggesting that MDT ability has a stronger impact on accuracy for nonMPs and vowelMPs compared to consMPs (see Table 1; hypotheses 13 and 15) was also unexpected, as we thought MDT would influence the learning of all pair types equally.

\section{DISCUSSION}

In this study, we tested whether music perception abilities impact the learning of novel word pairs in a CSWL paradigm that provides no explicit instruction during the learning phase. Overall, we found that participants were able to learn all novel word-object pairings regardless of the phonological overlap between the novel words, mostly replicating (albeit a little lower) previous reported results using the same online protocol (Escudero et al., 2021). That is, overall accuracy was comparable for novel words that had large phonological differences, forming non-minimal pairs (nonMPs), and for words that differed in a single consonant (consMPs) or a single vowel (vowelMPs). Regarding the relation between accuracy and music perception abilities, participants with average MPT and MDT had similar word learning scores across pair types, with performance for consMP probably being slightly lower than for the other pair types. Crucially, we found unexpected results for how MPT and MDT influenced 


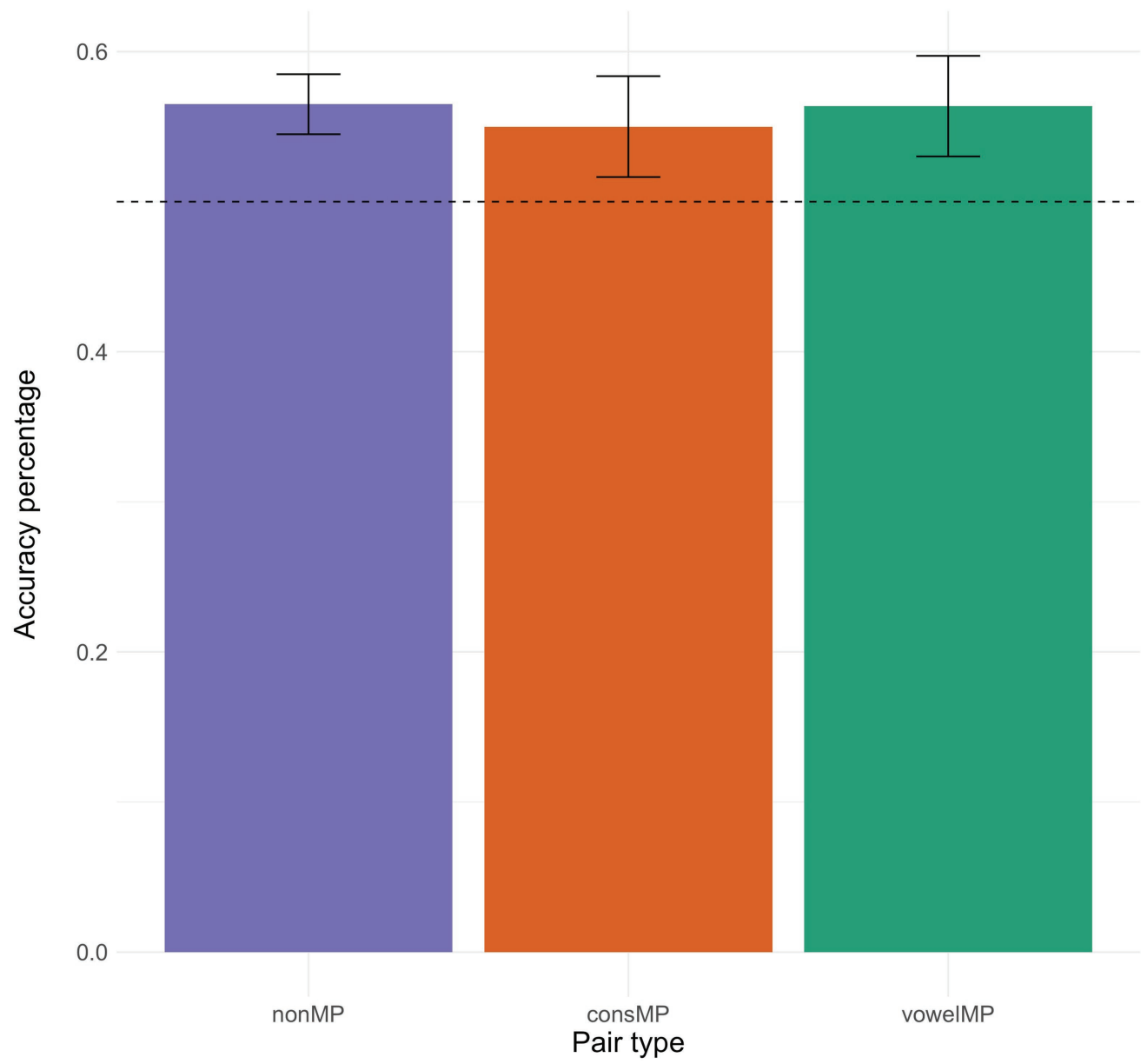

FIGURE 2 | Mean accuracy (in percentage) per pair type. Error bars represent the standard error over the mean accuracy responses per pair type. The dotted line represents accuracy by chance.

word learning performance in nonMPs versus consonant and vowelMPs, which we discuss below.

As mentioned above, although we expected higher MPT participants to learn vowel contrasts more easily due to the acoustic similarities between pitched musical sounds and vowels (consonants do not have a clear pitch), we found the opposite effect. It appears that stimuli containing variable pitch information (such as vowels) pose extra difficulty for listeners who are more attuned to such information. A plausible explanation for these results is proposed by Ong et al. (2017b) who suggest that listeners' experience is important for their ability to learn new acoustic cues, whether this experience is linguistic (through a native language that distinguishes lexical tone contrasts, such as Cantonese, Mandarin, or Thai) or musical. In a distributional learning (a form of statistical learning) experiment of nonnative lexical tones, they found that listeners without music or tonal language experience were able to discriminate lexical tones from ambiguous versions of the target tones after a short exposure (Ong et al., 2015). In a follow-up study, they found mixed results for pitch experts, who they define as listeners with extensive experience with pitch either through a tonal language or through musical training. Those with a tonal language background were able to learn non-native lexical tones distributionally but those with a musical background were not. This was unexpected as musical training has been found to have a positive effect on statistical learning (e.g., François et al., 2013; Chobert et al., 2014), and musicians were expected to perform better due to an improved ability to extract regularities from the input. These results led Ong and colleagues to conclude that domain-specific experience with pitch influences the ability to learn non-native lexical tones distributionally (Ong et al., 2017b), indicating no cross-domain transfer of music and linguistic abilities in distributional learning.

Ong and colleagues discussed their results in relation to the Second Language Perception (L2LP) model (Escudero, 2005; van Leussen and Escudero, 2015; Elvin and Escudero, 2019; Elvin et al., 2020, 2021; Yazawa et al., 2020), suggesting that the tonal language speakers only had to shift their category boundaries to the novel tonal categories, whereas the musicians had to create new categories, which is more difficult (Ong et al., 2017b). Another possible explanation is that musicians did not consider the stimuli as speech tones and thus may 
have processed them as musical stimuli resulting in them not learning the tonal categories (Ong et al., 2017b), but this argument assumes that musical pitch cannot be learned distributionally. In a different study, Ong et al. (2017a) tested

TABLE 1 | Hypothesis testing-accuracy model.

\begin{tabular}{|c|c|c|c|c|c|}
\hline Hypothesis tests & Estimate & $\begin{array}{l}\text { Est. } \\
\text { Error }\end{array}$ & {$[90 \% \mathrm{Cl}]$} & $\begin{array}{l}\text { Evid. } \\
\text { Ratio }\end{array}$ & $\begin{array}{l}\text { Post. } \\
\text { Prob }\end{array}$ \\
\hline \multicolumn{6}{|c|}{ For average MDT and MPT ability: } \\
\hline 1. nonMP-consMP >0 & -1.83 & 1.67 & {$[-4.88,0.25]$} & 11.11 & 0.92 \\
\hline 2. nonMP-vowelMP > 0 & -0.63 & 1.80 & {$[-3.90,1.21]$} & 0.64 & 0.39 \\
\hline 3. vowelMP-consMP >0 & 1.20 & 2.47 & {$[-2.63,4.87]$} & 3.27 & 3.27 \\
\hline \multicolumn{6}{|c|}{ MPT ability > 0 in the following conditions and contrasts: } \\
\hline 4. nonMP & 0.41 & 0.70 & {$[-0.50,1.76]$} & 2.47 & 0.71 \\
\hline 5. consMP & 2.97 & 1.48 & {$[0.80,5.55]$} & 91.78 & 0.99 \\
\hline 6. vowelMP & -0.88 & 0.85 & {$[-2.08,0.17]$} & 12.10 & 0.92 \\
\hline 7. consMP-nonMP & 2.55 & 1.53 & {$[0.28,5.20]$} & 30.61 & 0.97 \\
\hline 8. nonMP-vowelMP & 1.30 & 1.05 & {$[-0.07,3.04]$} & 16.37 & 0.94 \\
\hline 9. consMP-vowelMP & 3.85 & 1.69 & {$[1.38,6.68]$} & 92.75 & 0.99 \\
\hline \multicolumn{6}{|c|}{ MDT ability > 0 in the following conditions and contrasts: } \\
\hline 10. nonMP & 0.95 & 0.42 & {$[0.28,1.63]$} & 78.30 & 0.99 \\
\hline 11. consMP & -0.08 & 0.84 & {$[-1.45,1.11]$} & 0.92 & 0.48 \\
\hline 12. vowelMP & 1.16 & 0.93 & {$[-0.07,2.65]$} & 16.33 & 0.94 \\
\hline 13. nonMP-consMP & 1.04 & 0.89 & {$[-0.24,2.52]$} & 10.06 & 0.91 \\
\hline 14. vowelMP-nonMP & 0.21 & 0.98 & {$[-1.78,1.14]$} & 1.44 & 0.59 \\
\hline 15. vowelMP-consMP & 1.24 & 1.23 & {$[-0.54,3.29]$} & 7.63 & 0.88 \\
\hline
\end{tabular}

Estimate $=$ mean of the effect's posterior distribution. Estimate error $=$ standard deviation of the posterior distribution. $90 \% \mathrm{Cl}=90 \%$ credibility intervals. Evidence ratio $=$ the posterior probability under the hypothesis against its alternative. distributional learning of musical pitch with nonmusicians and showed that they were able to acquire pitch from a novel musical system in this manner. This may be different for musicians, who were found to outperform nonmusicians in the discrimination and identification of Cantonese lexical tones (Ong et al., 2020).

From studies on distributional learning of pitch and lexical tones, it can be concluded that cross-domain transfer between speech and music largely depends on the listener's musical or linguistic experience (Ong et al., 2015, 2016, 2017a,b, 2020). Nonmusicians without tonal language experience can learn novel pitch contrasts in both the speech and the music domain, but the situation is more complex for pitch experts, suggesting that those with extensive music experience may struggle more than those with tonal experience. However, an important difference between Ong et al's studies and the current study is that they tested listeners at both ends of the experience spectrum, while we tested listeners ranging from the lower to middle end of the music experience spectrum based on their music perception skills. By using music perception tasks, we were able to classify participants using a continuous predictor rather than splitting them into groups, which allowed us to uncover more detailed information about what happens with speech learning as music perception skills increase. A further difference is in the stimuli used, as the lexical and musical tones used in Ong et al. (2015, 2016, 2017a,b, 2020) contained many variable pitches along a continuum, while our stimuli had limited and uncontrolled pitch variation. Specifically, we focused

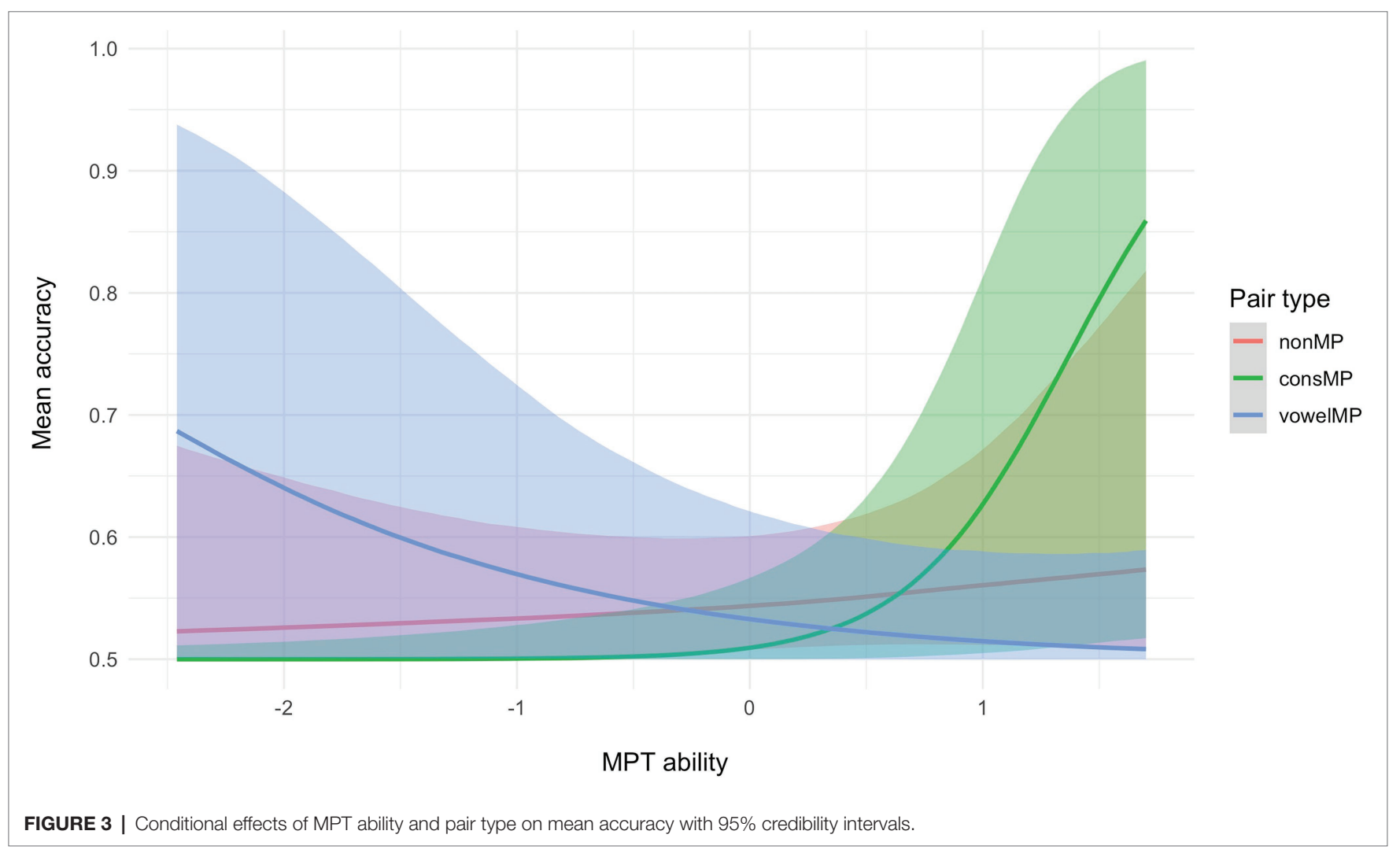




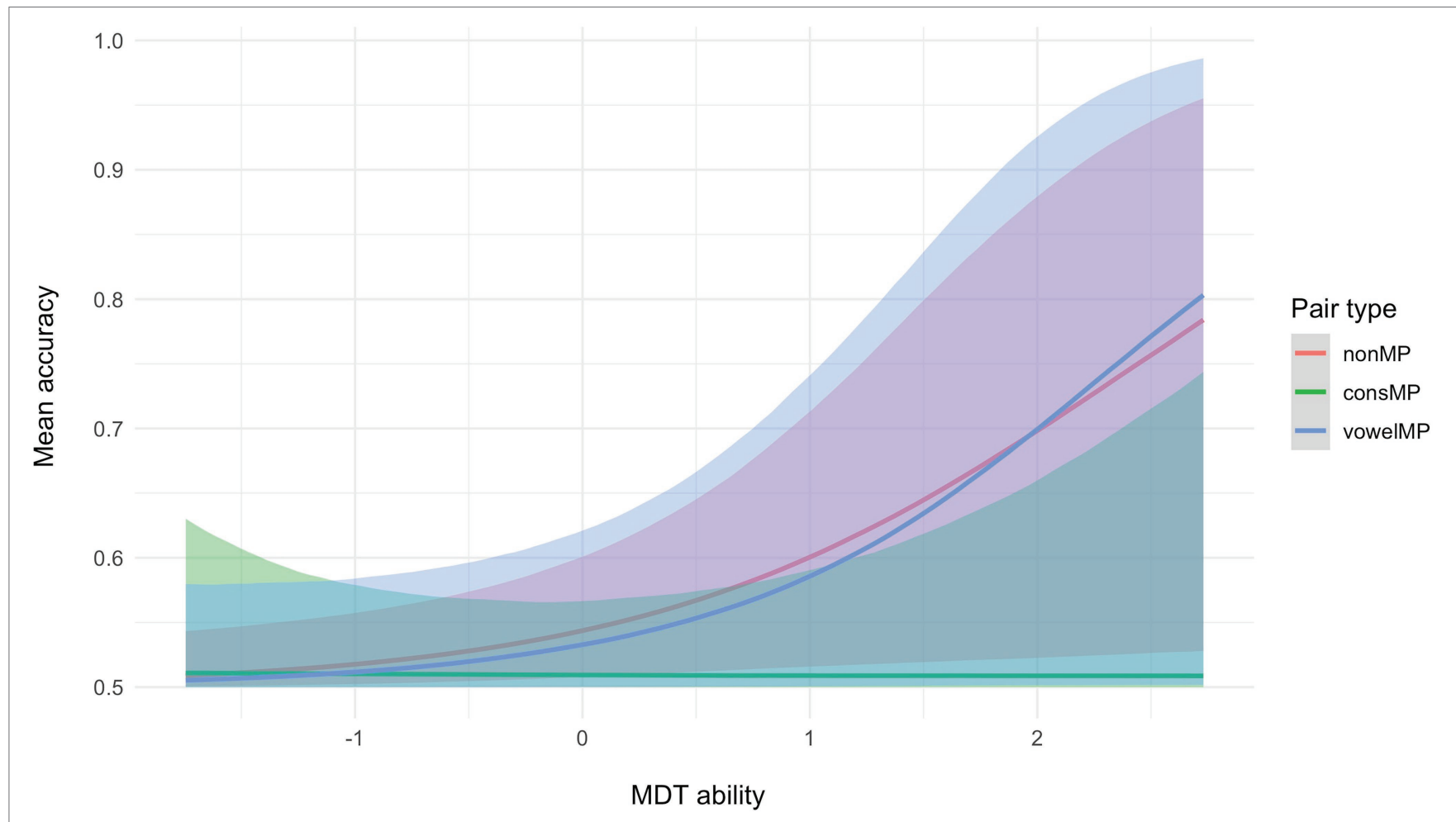

FIGURE 4 | Conditional effects of MDT ability and pair type on mean accuracy with 95\% credibility intervals.

on word learning of naturally produced novel words, where pitch variability was not consistent among the different words and pair types. Thus, listeners in the present study may have used other acoustic cues that are not pitch-related to discriminate and learn the novel words.

Given that listeners with strong pitch perception abilities are more likely to use pitch as a cue to discriminate between stimuli (Perfors and Ong, 2012; Ong et al., 2017b, 2020), our vowelMP stimuli may have been particularly challenging for them due to the use of infant-directed speech (IDS). IDS is the speech style or register typically used by mothers and caregivers when speaking to babies and is characterized by the use of larger pitch variations. Many studies have shown that IDS can facilitate word learning in infants (Ma et al., 2011; Graf Estes and Hurley, 2013) and adults (Golinkoff and Alioto, 1995) due to higher salience leading to enhanced attentional processing (Golinkoff and Alioto, 1995; Kuhl et al., 1997; Houston-Price and Law, 2013; Ellis, 2016). Despite it facilitating infant and adult speech learning, IDS may have a negative effect for those with strong musical perception abilities as they might think they are hearing different words due to varying pitch contours when only one word is presented. Unexpectedly, MPT ability affected learning of cMPS and nonMPs more than vMPs. As vMPs naturally contain more pitch variation, those were expected to be the most difficult to learn, hence the influence of IDS is likely stronger on cMPS and nonMPs than on vMPs. A similar result of hearing multiple words instead of one due to the use of IDS has been found in a prior CSWL study (Escudero et al., under review), where the target population consisted of native Mandarin speakers who were L2 English learners. Specifically, word pairs containing non-native vowel contrasts with IDS pitch fluctuations were difficult to learn for L1 Mandarin L2 English learners.

Thus, in populations where pitch variations indicate different lexical meanings, such as native speakers of Mandarin (Han, 2018), IDS can be problematic and impair word learning as participants might perceive multiple categories where only one is presented (Escudero and Boersma, 2002; Elvin et al., 2014; van Leussen and Escudero, 2015). The impact of a learner's native language on novel language learning has been explained by L2 speech theories (e.g., Flege, 1995; Escudero, 2005; Best and Tyler, 2007; van Leussen and Escudero, 2015). In particular, the L2LP model (Escudero, 2005; van Leussen and Escudero, 2015; Elvin and Escudero, 2019; Elvin et al., 2020, 2021; Yazawa et al., 2020) proposes three learning problems when L1 and L2 categories differ in number or in phonetic realization. This model is the only one that handles lexical development and word learning with consideration of hearing more differences than produced in the target language as a learning problem (van Leussen and Escudero, 2015; Escudero and Hayes-Harb, 2021). Specifically, listeners can categorize binary L2 contrasts into more than two L1 categories, which is referred to as Multiple Category Assimilation (MCA, L2LP; Escudero and Boersma, 2002) and can lead to a subset problem (Escudero and Boersma, 2002; Escudero, 2005; Elvin and Escudero, 2014, 2019). A subset problem occurs when an L2 category does not exist in a listener's L1 but is acoustically similar to two or more separate L1 categories and thus is perceived as more 
than one L1 sound, with no overt information from the target language that will allow the learner to stop hearing the extra category or stop activating irrelevant or spurious lexical items (Escudero and Boersma, 2002; Escudero, 2005; Elvin and Escudero, 2014, 2019).

With regard to our CSWL task, we expect that using adultdirected speech (ADS) without these additional pitch fluctuations would improve learning for the nonMPs and consMPs for tonal language speakers, but not for vowelMPs. When using IDS, nonmusicians and non-tonal speakers show a pattern where performance is lowest for pair types with the highest pitch variability (i.e., vowelMPs). The use of IDS, which adds even more pitch variability than naturally present in the vowelMPs, seems to pose problems for learners who are not music experts but have some music perception skills. For tonal language speakers, the use of IDS poses problems in general as they consistently use pitch information to discriminate between all pair types. If pitch variability is the main predictor for performance in this CSWL task, then music experts (i.e., musicians) should struggle more with the vowelMPs than the nonmusicians tested here but should perform better for the nonMPs and consMPs than the tonal language speakers discussed earlier in Escudero et al. (under review).

Regarding the results for MDT, although not decisive, the evidence suggests that MDT ability more strongly influences accuracy for nonMPs and vowelMPs compared to consMPs. The MDT ability test focuses heavily on auditory short-term memory (Dowling, 1978; Harrison et al., 2017; Harrison and Müllensiefen, 2018). It has been suggested that auditory short-term memory for consonants is distinct from that for vowels (Pisoni, 1975), as explained by the cue-duration hypothesis (Pisoni, 1973), which suggests that the acoustic features needed to discriminate between two different consonants are shorter and thus less well represented in auditory short-term memory than those of vowels (Chen et al., 2020). As well, seminal studies on speech sounds have suggested that consonants may be stored differently in short-term memory compared to vowels (Crowder, 1971, 1973a,b), with the idea that vowels are processed at an earlier stage compared to consonants (Crowder and Morton, 1969). It is possible that a different type of auditory memory is activated for nonMPs, which does not rely as strongly on the discrimination of the acoustic features of the stimuli than what is needed to distinguish between phonologically overlapping stimuli. As similarly suggested in Escudero et al. (2021), this could be tested using time-sensitive neurophysiological methods, such as electroencephalography (EEG).

Some limitations of this study must be noted. Even though we tested for perceptual skills, it is possible that accuracy also depends on other skills, such as how well a listener is able to do crossmodal associations. Likewise, it is possible that general cognitive abilities may impact the learning of novel words in an ambiguous word learning paradigm. As we find some differences between accuracy for the different pair types in the current study and prior CSWL studies using the same paradigm (Escudero et al., 2016; Mulak et al., 2019), it might seem that individual differences, such as the ability to do crossmodal associations or general cognitive abilities, may be the cause of these differences. However, there are other possible sources between the current study and prior CSWL results that might have led to the differences between studies, such as the number of trials and the number of responses used in the learning and test phases. We are currently replicating learning and testing phases from those previous studies using online testing to see if the number of trials is the source of the difference. If this is not the case, future studies can then look further into other possible sources, such as general cognitive abilities. Regarding the use of IDS, it is an empirical question whether adults in general will perform better with stimuli characterized by shorter durations, and non-enhanced differences between vowels and neutral prosodic contours (such as ADS). On the contrary, we found that enhanced vowel differences that are similar to those typical of IDS facilitate phonetic discrimination for adults listeners (Escudero et al., 2011; Escudero and Williams, 2014). Additionally, there is a possibility that the degree of novelty of the auditory and visual stimuli impacts accuracy responses. Even though language background did not have an influence on accuracy, future studies could consider implementing measuring participants' familiarity with the stimuli. Another possible limitation is that we did not collect information regarding participants' headphones. However, we did check whether participants were able to hear the stimuli and were wearing headphones, as part of our pre-registered protocol.

Overall, the results show that the tested music perception abilities impact the learning of words that differ in a single consonant or vowel differently and in complex ways. Pitch perception is an important factor for novel word learning, to the extent that those with stronger pitch perception skills are better at distinguishing consonant contrasts, and apparently too good at distinguishing vowel contrasts. Using stimuli produced in adult-directed-speech, our follow-up research will establish whether the negative correlation between pitch perception and accuracy in words distinguished by a single vowel is due to our use of IDS and its concomitant large pitch variations. We also find that consonants and vowels are learned differently for those with melodic discrimination skills, reflected in improved auditory short-term memory. In contrast to MPT, an increase in MDT leads to better learning of words distinguished by a single vowel than those distinguished by a single consonant, which may be connected to better auditory short-term memory for vowels. The contrasting results for the two tested music perception skills may reflect different stages of processing. Our results have one clear implication for theories of cross-domain transfer between music and language: considering populations along the entire spectrum of musicality and linguistic pitch experiences is the only way to uncover exactly where and when problems with word learning occur.

\section{CONCLUSION}

We tested whether specific music perception abilities impact learning of minimal pair types in adults that have not been selected for their musical abilities. Using a CSWL paradigm, 
we have shown that pitch perception and auditory working memory affect the learning of vowel and consonant minimal word pairs, but vowels and consonants are impacted differently. We suggest this may be due to the pitch fluctuations of the specific characteristic of stimuli, namely, words produced in infant-directed speech (IDS). Similar to the patterns observed in native speakers of tonal languages, this type of speech register may lead to the listeners' perception of more distinctions than intended. In future studies, we aim to test the role of IDS compared to adult-directed speech, how specific levels of training in music impact performance in CSWL, and the differential storage of vowels versus consonants.

\section{DATA AVAILABILITY STATEMENT}

The raw data supporting the conclusions of this article will be made available by the authors, without undue reservation.

\section{ETHICS STATEMENT}

The studies involving human participants were reviewed and approved by the Western Sydney University Human Research Ethics Committee (H11022). The participants provided their written informed consent to participate in this study.

\section{REFERENCES}

Agresti, A. (2010). Analysis of Ordinal Categorical Data. New York: John Wiley \& Sons.

Anvari, S. H., Trainor, L. J., Woodside, J., and Levy, B. A. (2002). Relations among musical skills, phonological processing, and early reading ability in pre-school children. J. Exp. Psychol. 83, 111-130. doi: 10.1016/ s0022-0965(02)00124-8

Barwick, J., Valentine, E., West, R., and Wilding, J. (1989). Relations between reading and musical abilities. Br. J. Educ. Psychol. 59, 253-257. doi: 10.1111/ j.2044-8279.1989.tb03097.x

Bergman Nutley, S., Darki, F., and Klingberg, T. (2014). Music practice is associated with development of working memory during childhood and adolescence. Front. Hum. Neurosci. 7:926. doi: 10.3389/fnhum.2013.00926

Besson, M., Chobert, J., and Marie, C. (2011). Transfer of training between music and speech: common processing, attention, and memory. Front. Psychol. 2:94. doi: 10.3389/fpsyg.2011.00094

Besson, M., Schön, D., Moreno, S., Santos, A., and Magne, C. (2007). Influence of musical expertise and musical training on pitch processing in music and language. Restor. Neurol. Neurosci. 25, 399-410. doi: 10.1371/journal.pone.0089642

Best, C. T., and Tyler, M. D. (2007). "Nonnative and second-language speech perception: commonalities and complementaries," in Language Experience in Second Language Speech Learning: In Honor of James Emil Flege. eds. O.-S. Bohn and M. J. Munro (Amsterdam: John Benjamins), 13-34.

Bidelman, G. M., and Alain, C. (2015). Musical training orchestrates coordinated neuroplasticity in auditory brainstem and cortex to counteract age-related declines in categorical vowel perception. J. Neurosci. 35, 1240-1249. doi: 10.1523/jNEUROSCIE.3292-14.2015

Bidelman, G. M., Hutka, S., and Moreno, S. (2013). Tone language speakers and musicians share enhanced perceptual and cognitive abilities for musical pitch: evidence for bidirectionality between the domains of language and music. PLoS One 8:e60676. doi: 10.1371/journal.pone.0060676

Bigand, E., and Poulin-Charronnat, B. (2006). Are we "experienced listeners"? A review of the musical capacities that do not depend on formal musical training. Cognition 100, 100-130. doi: 10.1016/j.cognition.2005.11.007

\section{AUTHOR CONTRIBUTIONS}

ES and PE conceived the initial experiments. ES was responsible for overseeing data collection and wrote the initial draft. ES and AM analyzed the data. ES, AM, and PE wrote the paper. All authors contributed to the article and approved the submitted version.

\section{FUNDING}

Data collection was funded by a Transdisciplinary \& Innovation Grant from the Australian Research Centre for the Dynamics of Language (project number TIG1112020) awarded to ES. PE's and ES' work and the article publication fees were funded by an Australian Research Council Future Fellowship (FT160100514) awarded to PE. AM's work was funded by an Australian Research Council Discovery Early Career Researcher Award (project number DE170100353).

\section{ACKNOWLEDGMENTS}

We would like to acknowledge and thank Deeahn Sako, Madeleine Leehy, and Christopher Piller for their help with data collection and the participants for their time and participation.

Bittner, R., Salamon, J., Tierney, M., Mauch, M., Cannam, C., and Bello, J. (2014). MedleyDB: a multitrack dataset for annotation-intensive MIR research. Paper presented at the International Society for Music Information Retrieval (ISMIR), Taipei, Taiwan.

Boebinger, D., Evans, S., Rosen, S., Lima, C. F., Manly, T., and Scott, S. K. (2015). Musicians and non-musicians are equally adept at perceiving masked speech. J. Acoust. Soc. Am. 137, 378-387. doi: 10.1121/1.4904537

Bürkner, P.-C. (2017). Brms: an R package for Bayesian multilevel models using Stan. J. Stat. Softw. 80, 1-28. doi: 10.18637/jss.v080.i01

Bürkner, P.-C. (2018). Advanced Bayesian multilevel modeling with the $\mathrm{R}$ package brms. R Journal 10, 395-411. doi: 10.32614/RJ-2018-017

Bürkner, P.-C. (2020). Bayesian Item Response Modeling in R with brms and Stan. arXiv [Epub ahead of print]

Burnham, D., Brooker, R., and Reid, A. (2015). The effects of absolute pitch ability and musical training on lexical tone perception. Psychol. Music 43, 881-897. doi: 10.1177/0305735614546359

Chandrasekaran, B., Krishnan, A., and Gandour, J. T. (2009). Relative influence of musical and linguistic experience on early cortical processing of pitch contours. Brain Lang. 108, 1-9. doi: 10.1016/j.bandl.2008.02.001

Chang, W., Cheng, J., Allaire, J. J., Xi, Y., and McPherson, J. (2020). Shiny: web application framework for R. Tech. Innov. Stat. Educ. 1:7492. doi: 10.5070/ T591027492

Chen, S., Zhu, Y., Wayland, R., and Yang, Y. (2020). How musical experience affects tone perception efficiency by musicians of tonal and non-tonal speakers? PLoS One 15:e0232514. doi: 10.1371/journal.pone.0232514

Chobert, J., Francois, C., Velay, J. L., and Besson, M. (2014). Twelve months of active musical training in 8- to 10-year-old children enhances the preattentive processing of syllabic duration and voice onset time. Cereb. Cortex 24, 956-967. doi: 10.1093/cercor/bhs377

Crowder, R. G. (1971). The sound of vowels and consonants in immediate memory. J. Verb. Learni. Behav. 10, 587-596. doi: 10.1016/S0022-5371(71)80063-4

Crowder, R. G. (1973a). Representation of speech sounds in precategorical acoustic storage. J. Exp. Psychol. 98, 14-24. doi: 10.1037/h0034286

Crowder, R. G. (1973b). Precategorical acoustic storage for vowels of short and long duration. Percept. Psychophys. 13, 502-506. doi: 10.3758/BF03205809 
Crowder, R. G., and Morton, J. (1969). Precategorical acoustic storage (PAS). Percept. Psychophys. 5, 365-373. doi: 10.3758/BF03210660

de Ayala, R. J. (2009). The Theory and Practice of Item Response Theory. New York, NY: The Guilford Press.

Degé, F., and Schwarzer, G. (2011). The effect of a music program on phonological awareness in preschoolers. Front. Psychol. 2:124. doi: 10.3389/fpsyg.2011.00124

Dittinger, E., Barbaroux, M., D’Imperio, M., Jäncke, L., Elmer, S., and Besson, M. (2016). Professional music training and novel word learning: from faster semantic encoding to longer-lasting word representations. J. Cogn. Neurosci. 28, 1584-1602. doi: 10.1162/jocn_a_00997

Dittinger, E., Chobert, J., Ziegler, J. C., and Besson, M. (2017). Fast brain plasticity during word learning in musically-trained children. Front. Hum. Neurosci. 11:233. doi: 10.3389/fnum.2017.00233

Dittinger, E., Scherer, J., Jäncke, L., Besson, M., and Elmer, S. (2019). Testing the influence of musical expertise on novel word learning across the lifespan using a cross-sectional approach in children, young adults and older adults. Brain Lang. 198:104678. doi: 10.1016/j.bandl.2019.104678

Dowling, W. J. (1978). Scale and contour: two components of a theory of memory for melodies. Psychol. Rev. 85, 341-354. doi: 10.1037/0033-295X.85.4.341

Ellis, N. C. (2016). Salience, cognition, language complexity, and complex adaptive systems. Stud. Second. Lang. Acquis. 38, 341-351. doi: 10.1017/ S027226311600005X

Elmer, S., Klein, C., Kühnis, J., Liem, F., Meyer, M., and Jäncke, L. (2014). Music and language expertise influence the categorization in musically trained and untrained subjects. Cereb. Cortex 22, 650-658. doi: 10.1093/cercor/bhr142

Elmer, S., Meyer, M., and Jäncke, L. (2012). Neurofunctional and behavioral correlates of phonetic and temporal categorization in musically trained and untrained subjects. Cereb. Cortex 22, 650-658. doi: 10.1093/cercor/bhr142

Elvin, J., and Escudero, P. (2014). "Perception of Brazilian Portuguese Vowels by Australian English and Spanish Listeners," in Proceedings of the International Symposium on the Acquisition of Second Language Speech Concordia Working Papers in Applied Linguistics. 5, 145-156.

Elvin, J., and Escudero, P. (2019). "Cross-linguistic influence in second language speech: implications for learning and teaching," in Cross-Linguistic Influence: From Empirical Evidence to Classroom Practice. eds. M. Juncal Gutierrez-Mangado, M. Martínez-Adrián and F. Gallardo-del-Puerto (Cham: Springer), 1-20.

Elvin, J., Escudero, P., and Vasiliev, P. (2014). Spanish is better than English for discriminating Portuguese vowels: acoustic similarity versus vowel inventory. Front. Psychol. 5:1188. doi: 10.3389/fpsyg.2014.01188

Elvin, J., Williams, D., and Escudero, P. (2020). "Learning to perceive, produce and recognise words in a non-native language," in Linguistic Approaches to Portuguese as an Additional Language. eds. K. V. Molsing, C. B. L. Perna and A. M. T. Ibaños (Amsterdam: John Benjamins Publishing Company).

Elvin, J., Williams, D., Shaw, J. A., Best, C. T., and Escudero, P. (2021). The role of acoustic similarity and non-native categorisation in predicting nonnative discrimination: Brazilian Portuguese vowels by English vs. Spanish listeners. Languages 6:44. doi: 10.3390/languages6010044

Escudero, P., Benders, T., and Wanrooij, K. (2011). Enhanced bimodal distributions facilitate the learning of second language vowels. J. Acoust. Soc. Am. 130, EL206-EL212. doi: 10.1121/1.3629144

Escudero, P., and Boersma, P. (2002). "The subset problem in L2 perceptual development: multiple- category assimilation by Dutch learners of Spanish." in Proceedings of the 26th Annual Boston University Conference on Language Development. eds. B. Skarabela, S. Fish, and A. H.-J. Do. November 2-4, 2001. Somerville, MA: Cascadilla Press, 208-219.

Escudero, P., and Hayes-Harb, R. (2021). The ontogenesis model may provide a useful guiding framework, but lacks explanatory power for the nature and development of L2 lexical representation. Biling. Lang. Congn. 1-2. doi: $10.1017 /$ S1366728921000602

Escudero, P., Mulak, K. E., Fu, C. S., and Singh, L. (2016a). More limitations to monolingualism: bilinguals outperform monolinguals in implicit word learning. Front. Psychol. 7:1218. doi: 10.3389/fpsyg.2016.01218

Escudero, P., Mulak, K. E., and Vlach, H. A. (2016b). Cross-situational word learning of minimal word pairs. Cogn. Sci. 40, 455-465. doi: 10.1111/ cogs. 12243

Escudero, P., Mulak, K. E., and Vlach, H. A. (2016c). Infants encode phonetic detail during cross-situational word learning. Front. Psychol. 7:1419. doi: 10.3389/fpsyg.2016.01419
Escudero, P., Smit, E. A., and Angwin, A. (2021). Investigating orthographic versus auditory cross-situational word learning with online and lab-based research. PsyArXive doi: 10.31234/osf.io/tpn5e [Epub ahead of print]

Escudero, P. (2005). Linguistic Perception and Second Language Acquisition: Explaining the Attainment of Optimal Phonological Categorization. LOT Dissertation Series 113, Utrecht University.

Escudero, P., and Williams, D. (2014). Distributional learning has immediate and long-lasting effects. Cognition 133, 408-413. doi: 10.1016/j. cognition.2014.07.002

Flege, J. E. (1995). Second Language Speech Learning Theory, Findings, and Problems.

François, C., Chobert, J., Besson, M., and Schön, D. (2013). Music training for the development of speech segmentation. Cereb. Cortex 23, 2038-2043. doi: 10.1093/cercor/bhs180

Gathercole, S. E., Hitch, G. J., and Marin, A. J. (1997). Phonological short-term memory and new word learning in children. Dev. Psychol. 33, 966-979. doi: 10.1037/0012-1649.33.6.966

Golinkoff, R. M., and Alioto, A. (1995). Infant-directed speech facilitates lexical learning in adults hearing Chinese: implications for language acquisition. J. Child Lang. 22, 703-726. doi: 10.1017/S0305000900010011

Gordon, R. L., Shivers, C. M., Wieland, E. A., Kotz, S. A., Yoder, P. J., and McAuley, J. D. (2015). Musical rhythm discrimination explains individual differences in grammar skills in children. Dev. Sci. 18, 635-644. doi: 10.1111/ desc. 12230

Graf Estes, K., and Hurley, K. (2013). Infant-directed prosody helps infants map sounds to meanings. Infancy 18, 797-824. doi: 10.1111/infa.12006

Hallam, S. (2017). The impact of making music on aural perception and language skills: a research synthesis. Lond. Rev. Educ. 15, 388-406. doi: 10.18546/ LRE.15.3.05

Han, M., de Jong, N. H., and Kager, R. (2018). Lexical tones in mandarin Chinese infant-directed speech: age-related changes in the second year of life. Front. Psychol. 9:434. doi: 10.3389/fpsyg.2018.00434

Hansen, M., Wallentin, M., and Vuust, P. (2012). Working memory and musical competence of musicians and nonmusicians. Psychol. Music 41, 779-793. doi: $10.1177 / 0305735612452186$

Harrison, P. M. C., Collins, T., and Müllensiefen, D. (2017). Applying modern psychometric techniques to melodic discrimination testing: item response theory, computerised adaptive testing, and automatic item generation. Sci. Rep. 7:3618. doi: 10.1038/s41598-017-03586-z

Harrison, P. M. C., and Müllensiefen, D. (2018). Melodic discrimination test (MDT), psychTestR implementation. Zenodo. doi: 10.5281/zenodo.1300950

Houston-Price, C., and Law, B. (2013). "How experiences with words supply all the tools in the toddler's word - learning toolbox," in Theoretical and Computational Models of Word Learning: Trends in Psychology and Artificial Intelligence. eds. L. Gogate and G. Hollich (Hershey, PA: IGI Global), 81-108.

Jeffreys, H. (1998). The Theory of Probability. England: OUP Oxford.

Jentschke, S., and Koelsch, S. (2009). Musical training modulates the development of syntax processing in children. NeuroImage 47, 735-744. doi: 10.1016/j. neuroimage.2009.04.090

Jonaitis, E. M., and Saffran, J. R. (2009). Learning harmony: the role of serial statistics. Cogn. Sci. 33, 951-968. doi: 10.1111/j.1551-6709.2009.01036.x

Kachergis, G., Yu, C., and Shiffrin, R. M. (2010). "Cross-situational statistical learning: implicit or intentional?" in Proceedings of the Annual Meeting of the Cognitive Science Society. Vol 32. August 11-14, 2010.

Kraus, N., and Chandrasekaran, B. (2010). Music training for the development of auditory skills. Nat. Rev. Neurosci. 11, 599-605. doi: 10.1038/nrn2882

Kraus, N., and White-Schwoch, T. (2017). Neurobiology of everyday communication: what have we learned from music? Neuroscientist 23, 287-298. doi: $10.1177 / 1073858416653593$

Krishnan, A., Xu, Y., Gandour, J., and Cariani, P. (2005). Encoding of pitch in the human brainstem is sensitive to language experience. Cogn. Brain Res. 25, 161-168. doi: 10.1016/j.cogbrainres.2005.05.004

Krizman, J., Marian, V., Shook, A., Skoe, E., and Kraus, N. (2012). Subcortical encoding of sound in enhanced in bilinguals and relates to executive function advantages. Proc. Natl. Acad. Sci. 109, 7877-7881. doi: 10.1073/ pnas. 1201575109

Kruschke, J. K. (2018). Rejecting or accepting parameter values in Bayesian estimation. Adv. Methods Pract. Psychol. Sci. 1, 270-280. doi: $10.1177 / 2515245918771304$ 
Kuhl, P. K., Andruski, J. E., Chistovich, I. A., Chistovich, L. A., Kozhevnikova, E. V., Ryskina, V. L., et al. (1997). Cross-language analysis of phonetic units in language addressed to infants. Science 277, 684-686. doi: 10.1126/ science. 277.5326 .684

Kühnis, J., Elmer, S., Meyer, M., and Jäncke, L. (2013). The encoding of vowels and temporal speech cues in the auditory cortex of professional musicians: an EEG study. Neuropsychologia 51, 1608-1618. doi: 10.1016/j. neuropsychologia.2013.04.007

Kunert, R., Willems, R. M., and Hagoort, P. (2016). An independent psychometric evaluation of the PROMS measure of music perception skills. PLoS One 11:e0159103. doi: 10.1371/journal.pone.0159103

Lamb, S. J., and Gregory, A. H. (1993). The relationship between music and reading in beginning readers. J. Educ. Psychol. 13, 19-27. doi: 10.1080/0144341930130103

Larrouy-Maestri, P., Harrison, P. M. C., and Müllensiefen, D. (2018). Mistuning perception test, psychTestR implementation. Zenodo. doi: 10.5281/ zenodo. 1415363

Larrouy-Maestri, P., Harrison, P. M. C., and Müllensiefen, D. (2019). The mistuning perception test: A new measurement instrument. Behav. Res. Methods 51, 663-675. doi: 10.3578/s13428-019-01225-1

Law, L. N. C., and Zentner, M. (2012). Assessing musical abilities objectively: construction and validation of the profile of music perception skills. PLoS One 7:e52508. doi: 10.1371/journal.pone.0052508

Leung, Y., and Dean, R. T. (2018). Learning unfamiliar pitch intervals: a novel paradigm for demonstrating the learning of statistical associations between musical pitches. PLoS One 13:e0203026. doi: 10.1371/journal.pone.0203026

Liu, J., Hilton, C. B., Bergelson, E., and Mehr, S. A. (2021). Language experience shapes music processing across 40 tonal, pitch-accented, and non-tonal languages. bioRxiv. doi: 10.1101/2021.10.18.464888 [Epub ahead of print]

Loui, P., Wessel, D. L., and Hudson Kam, C. L. (2010). Human rapidly learn grammatical structure in a new musical scale. Music. Percept. 27, 377-388. doi: $10.1525 / \mathrm{mp} .2010 .27 .5 .377$

Ma, W., Golinkoff, R. M., Houston, D. M., and Hirsh-Pasek, K. (2011). Word learning in infant- and adult-directed speech. Lang. Learn. Dev. 7, 185-201. doi: $10.1080 / 15475441.2011 .579839$

Magis, D., and Raîche, G. (2012). Random generation of response patterns under computerized adaptive testing with the R package catR. J. Stat. Softw. 48:i08. doi: 10.18637/jss.v048.i08

Magne, C., Schön, D., and Besson, M. (2006). Musician children detect pitch violations in both music and language better than nonmusician children: behavioral and electrophysiological approaches. J. Cogn. Neurosci. 18, 199-211. doi: 10.1162/jocn.2006.18.2.199

Makowski, D., Ben-Shachar, M. S., Chen, S. H. A., and Lüdecke, D. (2019). Indices of effect existence and significance in the Bayesian framework. Front. Psychol. 10:2767. doi: 10.3389/fpsyg.2019.02767

Marie, C., Delogu, F., Lampis, G., Belardinelli, M. O., and Besson, M. (2011a). Influence of musical expertise on segmental and tonal processing in mandarin Chinese. J. Cogn. Neurosci. 23, 2701-2715. doi: 10.1162/jocn.2010.21585

Marie, C., Magne, C., and Besson, M. (2011b). Musicians and the metric structure of words. J. Cogn. Neurosci. 23, 294-305. doi: 10.1162/jocn.2010.21413

Milne, A. J., and Herff, S. A. (2020). The perceptual relevance of balance, evenness, and entropy in musical rhythms. Cognition 203:104233. doi: 10.1016/j.cognition.2020.104233

Moreno, S., Marques, C., Santos, A., Santos, M., Castro, S. L., and Besson, M. (2009). Musical training influences linguistic abilities in 8-year-old children: more evidence for brain plasticity. Cereb. Cortex 19, 712-723. doi: 10.1093/cercor/bhn120

Mulak, K. E., Vlach, H. A., and Escudero, P. (2019). Cross-situational learning of phonologically overlapping words across degrees of ambiguity. Cogn. Sci. 43:e12731. doi: 10.1111/cogs.12731

Müllensiefen, D., Gingras, B., Musil, J., and Stewart, L. (2014). The musicality of non-musicians: an index for assessing musical sophistication in the general population. PLoS One 9:e101091. doi: 10.1371/journal.pone.0089642

Musacchia, G., Sams, M., Skoe, E., and Kraus, N. (2007). Musicians have enhanced subcortical auditory and audiovisual processing of speech and music. Proc. Natl. Acad. Sci. U. S. A. 104, 15894-15898. doi: 10.1073/ pnas.0701498104

Ong, J. H., Burnham, D., and Escudero, P. (2015). Distributional learning of lexical tones: a comparison of attended vs. unattended listening. PLoS One 10:e0133446. doi: 10.1371/journal.pone.0133446
Ong, J. H., Burnham, D., Escudero, P., and Stevens, C. J. (2017b). Effect of linguistic and musical experience on distributional learning of nonnative lexical tones. J. Speech Lang. Hear. Res. 60, 2769-2780. doi: 10.1044/2016_ JSLHR-S-16-0080

Ong, J. H., Burnham, D., and Stevens, C. J. (2017a). Learning novel musical pitch via distributional learning. J. Exp. Psychol. Learn. Mem. Cogn. 43, 150-157. doi: $10.1037 / x \operatorname{lm} 0000286$

Ong, J. H., Burnham, D., Stevens, C. J., and Escudero, P. (2016). Naïve learners show cross-domain transfer after distributional learning: the case of lexical and musical pitch. Front. Psychol. 7:1189. doi: 10.3389/fpsyg.2016.01189

Ong, J. H., Wong, P. C. M., and Liu, F. (2020). Musicians show enhanced perception, but not production, of native lexical tones. J. Acoust. Soc. Am. 148, 3443-3454. doi: 10.1121/10.0002776

Ott, C. G. M., Lnager, N., Oeschlin, M. S., Meyer, M., and Jäncke, L. (2011). Processing of voiced and unvoiced acoustic stimuli in musicians. Front. Psychol. 2:195. doi: 10.3389/fpsyg.2011.00195

Overy, K. (2003). Dyslexia and music: from timing deficits to musical intervention. Ann. N. Y. Acad. Sci. 999, 497-505. doi: 10.1196/ annals. 1284.060

Parbery-Clark, A., Strait, D. L., Anderson, S., Hittner, E., and Kraus, N. (2011). Musical experience and the aging auditory system: implications for cognitive abilities and hearing speech in noise. PLoS One 6:e18082. doi: 10.1371/ journal.pone.0018082

Patel, A. D. (2003). Language, music, syntax and the brain. Nat. Neurosci. 6, 674-681. doi: 10.1038/nn1082

Patel, A. D., and Iversen, J. R. (2007). The linguistic benefits of musical abilities. Trends Cogn. Sci. 11, 369-372. doi: 10.1016/j.tics.2007.08.003

Pearce, M. T., Ruiz, M. H., Kapasi, S., Wiggins, G. A., and Bhattacharya, J. (2010). Unsupervised statistical learning underpins computational, behavioural, and neural manifestations of musical expectation. NeuroImage 50, 302-313. doi: 10.1016/j.neuroimage.2009.12.019

Peirce, J. W. (2007). PsychoPy-psychophysics software in python. J. Neurosci. Methods 162, 8-13. doi: 10.1016/j.jneumeth.2006.11.017

Peirce, J. W., Gray, J. R., Simpson, S., MacAskill, M. R., Höchenberger, R., Sogo, H., et al. (2019). PsychoPy2: experiments in behavior made easy. Behav. Res. Methods 51, 195-203. doi: 10.3758/s13428-01801193-y

Perfors, A., and Ong, J. H. (2012). "Musicians Are Better at Learning Non-native Sound Contrasts Even in Non-tonal Languages," in Proceedings of the 34th Annual Conference of the Cognitive Science Society. eds. N. Miyake, D. Peebles and R. P. Cooper. August 1-4, 2012. Cognitive Science Society, 839-844.

Pinheiro, A. P., Vasconcelos, M., Dias, M., Arrais, N., and Gonçalves, Ó. F. (2015). The music of language: An ERP investigation of the effects of musical training on emotional prosody processing. Brain Lang. 140, 24-34. doi: 10.1016/j.bandl.2014.10.009

Pinker, S. (1997). How the Mind Works. New York: Norton.

Pisoni, D. B. (1973). Auditory and phonetic memory codes in the discrimination of consonants and vowels. Percept. Psychophys. 13, 253-260. doi: 10.3758/BF03214136

Pisoni, D. B. (1975). Auditory short-term memory and vowel perception. Mem. Cogn. 3, 7-18. doi: 10.3758/BF03198202

R Core Team (2020). R: A Language and Environment for Statistical Computing [Computer Software Manual]. Vienna, Austria. Available at: https://www.Rproject.org/

Rogalsky, C., Rong, F., Saberi, K., and Hickok, G. (2011). Functional anatomy of language and music perception: temporal and structural factors investigated using functional magnetic resonance imaging. J. Neurosci. 31, 3843-3852. doi: 10.1523/JNEUROSCI.4515-10.2011

Ruggles, D. R., Freyman, R. L., and Oxenham, A. J. (2014). Influence of musical training on understanding voiced and whispered speech in noise. PLoS One 9:e86980. doi: 10.1371/journal.pone.0086980

Sammler, D., Grigutsch, M., Fritz, T., and Koelsch, S. (2007). Music and emotion: electrophysiological correlates of the processing of pleasant and unpleasant music. Psychophysiology 44, 293-304. doi: 10.1111/j.1469-8986.2007.00497.x

Schön, D., Magne, C., and Besson, M. (2004). The music of speech: music training facilitates pitch processing in both music and language. Psychophysiology 41, 341-349. doi: 10.1111/1469-8986.00172.x

Schulze, K., Zysset, S., Mueller, K., Friederici, A. D., and Koelsch, S. (2011). Neuroarchitecture of verbal and tonal working memory in nonmusicians and musicians. Hum. Brain Mapp. 32, 771-783. doi: 10.1002/hbm.21060 
Slevc, L. R., and Miyake, A. (2006). Individual differences in second-language proficiency: does musical ability matter? Psychol. Sci. 17, 675-681. doi: 10.1111/j.1467-9280.2006.01765.x

Smit, E. A., Milne, A. J., Dean, R. T., and Weidemann, G. (2019). Perception of affect in unfamiliar musical chords. PLoS One 14:e0218570. doi: 10.1371/ journal.pone.0218570

Smith, A. D. M., and Smith, K. (2012). "Cross-situational learning," in Encyclopedia of the Sciences of Learning. ed. N. M. Seel (Boston, MA: Springer US), $864-866$.

Smith, L. B., and Yu, C. (2008). Infants rapidly learn word-referent mappings via cross-situational statistics. Cognition 106, 1558-1568. doi: 10.1016/j. cognition.2007.06.010

Stewart, E. C., and Pittman, A. L. (2021). Learning and retention of novel words in musicians and nonmusicians. J. Speech Lang. Hear. Res. 64, 2870-2884. doi: 10.1044/2021_JSLHR-20-00482

Strait, D. L., and Kraus, N. (2011a). Can you hear me now? Musical training shapes functional brain networks for selective auditory attention and hearing speech in noise. Front. Psychol. 2:113. doi: 10.3389/fpsyg.2011.00113

Strait, D. L., and Kraus, N. (2011b). Playing music for a smarter ear: cognitive, perceptual and neurobiological evidence. Music. Percept. 29, 133-146. doi: 10.1525/mp.2011.29.2.133

Strange, W. (ed.) (1999). "Second language speech learning theory, findings, and problems," in Speech perception and linguistic experience: issues in crosslanguage research. Timonium, MD: York Press, 229-273.

Suanda, S. H., Mugwanya, N., and Namy, L. L. (2014). Cross-situational statistical word learning in young children. J. Exp. Child Psychol. 126, 395-411. doi: 10.1016/j.jecp.2014.06.003

Swaminathan, S., and Gopinath, J. K. (2013). Music training and second-language English comprehension and vocabulary skills in Indian children. Psychol. Stud. 58, 164-170. doi: 10.1007/s12646-013-0180-3

Swaminathan, S., and Schellenberg, E. G. (2017). Musical competence and phoneme perception in a foreign language. Psychon. Bull. Rev. 24, 1929-1934. doi: 10.3758/s13423-017-1244-5

Talamini, F., Altoè, G., Carretti, B., and Grassi, M. (2017). Musicians have better memory than nonmusicians: a meta-analysis. PLoS One 12:e186773. doi: 10.1371/journal.pone.0186773

Talamini, F., Grassi, M., Toffalini, E., Santoni, R., and Carretti, B. (2018). Learning a second language: can music aptitude or music training have a role? Learn. Individ. Differ. 64, 1-7. doi: 10.1016/j.lindif.2018.04.003

Tallal, P., and Gaab, N. (2006). Dynamic auditory processing, musical experience and language development. Trends Neurosci. 29, 382-390. doi: 10.1016/j. tins.2006.06.003

Tervaniemi, M. (2001). "Musical sound processing in the human brain: evidence from electric and magnetic recordings," in The Biological Foundations of Music. Vol. 930. eds. R. J. Zatorre and I. Peretz (New York, NY: New York Academy of Sciences), 259-272.

Tervaniemi, M., Kujala, A., Alho, K., Virtanen, J., Ilmoniemi, R. J., and Näätänen, R. (1999). Functional specialization of the human auditory cortex in processing phonetic and musical sounds: A magnetoencephalographic (MEG) study. NeuroImage 9, 330-336. doi: 10.1006/nimg.1999.0405

Thomson, J. M., Leong, V., and Goswami, U. (2013). Auditory processing interventions and developmental dyslexia: A comparison of phonemic and rhythmic approaches. Read. Writ. 26, 139-161. doi: 10.1007/s11145-0129359-6

Tuninetti, A., Mulak, K., and Escudero, P. (2020). Cross-situational word learning in two foreign languages: effects of native and perceptual difficulty. Front. Commun. 5:602471. doi: 10.3389/fcomm.2020.602471 van Leussen, J.-W., and Escudero, P. (2015). Learning to perceive and recognize a second language: The L2LP model revised. Front. Psychol. 6:1000. doi: 10.3389/fpsyg.2015.01000

Varnet, L., Wang, T., Peter, C., Meunier, F., and Hoen, M. (2015). How musical expertise shapes speech perception: evidence from auditory classification images. Sci. Rep. 5:14489. doi: 10.1038/srep14489

Vlach, H. A., and Johnson, S. P. (2013). Memory constraints on infants' crosssituational statistical learning. Cognition 127, 375-382. doi: 10.1016/j. cognition.2013.02.015

Vlach, H. A., and Sandhofer, C. M. (2014). Retrieval dynamics and retention in cross-situational statistical word learning. Cogn. Sci. 38, 757-774. doi: $10.1111 /$ cogs. 12092

Warm, T. A. (1989). Weighted likelihood estimation of ability in item response theory. Psychometrika 54, 427-450. doi: 10.1007/BF02294627

Warmington, M. A., Kandru-Pothineni, S., and Hitch, G. J. (2019). Novel-word learning, executive control and working memory: a bilingual advantage Bilingualism 22, 763-782. doi: 10.1017/S136672891800041X

White-Schwoch, T., Carr, K. W., Anderson, S., Strait, D. L., and Kraus, N. (2013). Older adults benefit from music training early in life: Biological evidence for long-term training-driven plasticity. J. Neurosci. 33, 17667-17674. doi: 10.1523/JNEUROSCI.2560-13.2013

Wong, P. C. M., and Perrachione, T. K. (2007). Learning pitch patterns in lexical identification by native English-speaking adults. Appl. Psycholinguist. 28, 565-585. doi: 10.1017/S0142716407070312

Yang, H., Ma, W., Gong, D., Hu, J., and Yao, D. (2014). A longitudinal study on children's music training experience and academic development. Sci. Rep. 4:5854. doi: 10.1038/srep05854

Yazawa, K., Whang, J., Kondo, M., and Escudero, P. (2020). Language-dependent cue weighting: An investigation of perception modes in L2 learning. Second. Lang. Res. 36, 557-581. doi: 10.1177/0267658319832645

Yu, C., and Smith, L. B. (2007). Rapid word learning under uncertainty via cross-situational statistics. Psychol. Sci. 18, 414-420. doi: 10.1111/j.1467-9280.2007.01915.x

Zhu, J., Chen, X., and Yang, Y. (2021). Effects of amateur musical experience on categorical perception of lexical tones by native Chinese adults: an ERP study. Front. Psychol. 12:611189. doi: 10.3389/fpsyg.2021.611189

Zuk, J., Ozernov-Palchik, O., Kim, H., Lakshminarayanan, K., Gabrieli, J. D. E., Tallal, P., et al. (2013). Enhanced syllable discrimination thresholds in musicians. PLoS One 8:e80546. doi: 10.1371/journal.pone.0080546

Conflict of Interest: The authors declare that the research was conducted in the absence of any commercial or financial relationships that could be construed as a potential conflict of interest.

Publisher's Note: All claims expressed in this article are solely those of the authors and do not necessarily represent those of their affiliated organizations, or those of the publisher, the editors and the reviewers. Any product that may be evaluated in this article, or claim that may be made by its manufacturer, is not guaranteed or endorsed by the publisher.

Copyright (c) 2022 Smit, Milne and Escudero. This is an open-access article distributed under the terms of the Creative Commons Attribution License (CC BY). The use, distribution or reproduction in other forums is permitted, provided the original author(s) and the copyright owner(s) are credited and that the original publication in this journal is cited, in accordance with accepted academic practice. No use, distribution or reproduction is permitted which does not comply with these terms. 